Table 1. Overview of patients' perspectives on ULT treatment strategies

\begin{tabular}{|c|c|}
\hline Motivation for a T2T strategy & Drawbacks of a T2T strategy \\
\hline 1. Being free of flares & 1. Resistance to (any) medication use \\
\hline \multirow{2}{*}{$\begin{array}{l}\text { 2. Acceptance of and contentment with chronic } \\
\text { ULT use }\end{array}$} & 2. Side effects of ULT \\
\hline & 3. Possibly detrimental to healthcare costs \\
\hline \multicolumn{2}{|l|}{ 3. Feels secure due to regular SU monitoring } \\
\hline \multicolumn{2}{|l|}{ 4. No desire for change } \\
\hline Motivation for a T2S strategy & Drawbacks of a T2S strategy \\
\hline $\begin{array}{l}\text { 1. Doubt if chronic ULT use is necessary } \\
\text { a. Possible restorative capacity of the body } \\
\text { b. Curious to effects of ULT cessation }\end{array}$ & $\begin{array}{l}\text { 1. Fear and insecurities on } \\
\text { a. Flaring and not being able to function } \\
\text { b. Joint damage }\end{array}$ \\
\hline 2. Being free of ULT side effects & 2. Feels uncontrolled \\
\hline 3. Long term damage ULT unknown & 3. Hassle with visits, blood tests and \\
\hline 4. Less burdensome for patient and body & medication adjustments when a flare \\
\hline 5. Wish for minimization of (any) medication use & occurs. \\
\hline
\end{tabular}

Conclusion: This study provides an overview of perspectives on ULT treatment strategies of gout patients in remission. These results must be considered in developing educational material for patients and in future research on gout management, particularly in designing randomised clinical trials on this subject. REFERENCES:

[1] Richette P et al. Ann Rheum Dis. 2017;76(1):29-42.

[2] FitzGerald JD et al. Arthritis Care Res (Hoboken). 2020;72(6):744-60.

[3] Pope C et al. Qual Saf Health Care. 2002;11(2):148-52.

[4] de Lautour H et al. Arthritis Care Res (Hoboken). 2016;68(5):667-72.

[5] Pope C et al. Bmj. 2000;320(7227):114-6.

Acknowledgements: We would like to thank dr. Erik Bischoff for his cooperation and help in including primary care patients from his general practice UGC Heyendael, Nijmegen the Netherlands.

Disclosure of Interests: None declared

DOI: 10.1136/annrheumdis-2021-eular.890

\section{POS1478-HPR NURSE LED REMOTE MANAGEMENT OF OSTEOPOROSIS - DO PATIENTS APPROVE?}

J. Begum ${ }^{1}$, J. Fourmy ${ }^{1}$, M. K. Nisar ${ }^{1} .{ }^{1}$ Luton and Dunstable University Hospital, Rheumatology, Luton, United Kingdom

Background: Specialist services are heavily reliant on consultant delivered models however lack of sufficient consultant appointments pose a significant operational challenge. Clinical nurse specialists (CNSs) are highly trained and can fill this gap whilst maintaining a high standard of care.

Objectives: We designed an innovative proof-of-concept osteoporosis service with patients only consulting a metabolic bone CNS and a consultant providing remote oversight. The aim of the project was to improve the efficiency of the service by eliminating consultant appointments and reducing unnecessary hospital visits whilst continuing to deliver high-quality care.

Methods: A new pathway was implemented where a consultant rheumatologist and a CNS virtually triaged women over the age of 65 into the service. A dedicated proforma provided the template for the CNS to undertake new patient telephone consultation. Relevant investigations were requested during the telephone clinic and treatment related information was despatched to help with shared decision making. All patients were then reviewed in a consultant-CNS virtual MDT. Appropriate parenteral treatment option was agreed and confirmed to each individual. The CNS worked through a safety checklist and provided further advice and support to the patient as necessary. We surveyed 100 consecutive patients to gain their feedback regarding the service using a ten-item questionnaire employing binary and Likert scale options.

Results: The questionnaire was posted to 100 patients. Mean age of participants was 77.2 years (65-92). 75 returned the survey. 67 (89\%) were happy with the waiting time and seventy (93\%) found thirty-minute appointment acceptable. 66 (88\%) were happy with the phone consultation and agreed that the care plan was adequately explained. 73 (97\%) were happy for the CNS review in future and would recommend the service. 67 (89\%) were satisfied with the overall service. Though most (95\%) were able to take the call maintaining privacy, eleven (15\%) participants would've found physical appointment more convenient. When asked which medium they would prefer for future reviews, only fifty $(66 \%)$ patients would keep telephone as their choice. Sixteen (21\%) would prefer face to face consultation, seven (9\%) a combination of the two and only two would choose online option.

Conclusion: To our knowledge, this is the first successful example of an innovative service wholly provided by CNSs. Most patients were satisfied with this model of care and would highly recommend it. They were happy to be reviewed by CNS in the future. However a significant minority did not like the remote element and a higher proportion would choose physical review in future if offered. Interestingly, telephone consultation still scored substantially higher than online platform despite being physically visible in the latter. This is intriguing as most remote services strive for online consultations assuming its preference to phone. Our study suggests against this notion certainly in older patient group. Overall, a nurse-delivered osteoporosis treatment pathway is highly effective, safe and provides an innovative solution though remote consultations may not be the optimal delivery model.

Disclosure of Interests: None declared

DOI: 10.1136/annrheumdis-2021-eular.1072

\section{POS1479-HPR PATIENTS' PERCEPTIONS OF PERSON-CENTRED CARE IN EARLY RA: A QUALITATIVE STUDY}

E. Landgren $^{1,2,3}$, A. Bremander ${ }^{1,3,4,5}$, E. Lindqvist ${ }^{1,2}$, M. Nylander ${ }^{3,6}$,

I. Larsson ${ }^{1,3,7}$. ${ }^{1}$ Lunds University, Department of Clinical Sciences, Section of Rheumatology, Lund, Sweden; ${ }^{2}$ Skåne University Hospital, Department of Rheumatology, Lund, Sweden; ${ }^{3}$ Spenshult Research and Development Centre, Halmstad, Sweden; ${ }^{4}$ University of Southern Denmark, Department of Regional Health Research, Odense, Denmark; ${ }^{5}$ University Hospital of Southern Denmark, Danish Hospital for Rheumatic Diseases, Sonderborg, Denmark; ${ }^{6}$ Swedish Rheumatism Association, Stockholm, Sweden; ${ }^{7}$ Halmstad University, School of Health and Welfare, Halmstad, Sweden

Background: Data on patients' experience can identify strengths and weaknesses with given care, why a person-centred care will improve health care quality. In rheumatology care, most research on patient preferences for and experiences of RA care is performed in patients with established RA and less often in patients with early RA. In the early course of RA patients often struggle to manage their new life situation with a chronic disease and its treatment. Expectations and experiences of health care may change over time why it is important to understand how newly diagnosed patients perceive person-centred care.

Objectives: To explore patients' perceptions of person-centred care early in the RA disease course within the framework of person-centred care.

Methods: In this qualitative study 31 patients with early RA from four rheumatology specialist outpatient clinics were interviewed. An abductive qualitative content analysis was conducted based on the framework of McCormack and McCance $(2006,2016)$. The four constructs; prerequisites, care environment, person-centred processes, and person-centred outcomes constituted the four categories in the deductive part of the study. An inductive analysis revealed eleven sub-categories exploring the content of person-centred care for patients with early RA.

Results: For patients with early RA person-centred care was described as; 1 Prerequisites were to be treated with respect, to meet dedicated healthcare professionals, and to meet professional competence. 2. The care environment was to have access to a multidisciplinary team, to have access to health care, and a supportive organization. 3. Person-centred processes were to be listened to, to be supported, and to be involved in decision-making. 4. The person-centred outcomes were to be satisfied with received health care and to achieve optimal health.

Conclusion: A true person-centred care is important to patients early in the RA disease course, supporting the relevance to implement person-centred approach at all stages in the health care system. This study contributes to information about how to further develop person-centredness in rheumatology care also early in the disease course.

\section{REFERENCES:}

[1] McCormack, B., \& McCance, T. V. (2006). Development of a framework for person-centred nursing. $J$ Adv Nurs, 56(5), 472-479. doi:10.1111/j.1365-2648.2006.04042.x

[2] McCormack, B., \& McCance, T. V. (2016). Person-centred practice in nursing and health care: theory and practice (2nd edition ed.): John Wiley \& Sons.

Disclosure of Interests: None declared

DOI: 10.1136/annrheumdis-2021-eular.1240

\section{POS1480-HPR IMPLEMENTATION OF TELEMEDICINE IN RHEUMATOLOGY: VIRTUAL CONSULTATION AS A TOOL FOR DIRECT COMMUNICATION WITH PRIMARY CARE}

I. Garcia Hernandez ${ }^{1}$, D. V. Mendoza Mendoza ${ }^{1}$, P. Muñoz Reinoso ${ }^{1}$, J. J. Pérez Venegas ${ }^{1}$. ${ }^{1}$ Hospital Universitario Virgen Macarena, Rheumatology, Sevilla, Spain

Background: Virtual consultation is defined as the provision of a healthcare service when there is a distance between the subjects and information and communication technologies are used to carry out the consultation. This tool has been successfully implemented in different specialties. It is useful for providing quick solutions, improving the overload of the medical care and for the early detection of inflammatory diseases ${ }^{1}$. In our centre, virtual consultation from Primary Care (PC) to Hospital Care $(\mathrm{HC})$ has been implemented Objectives: The main objective is to describe our experience with the use of virtual consultation and its value as a new modality of specialised medical care. 
The second aim is to identify the most frequent reasons for consultation and diagnoses, to assess the concordance between the two and to analyse the trend over time of the number of virtual consultations and their relationship with the different waves of the COVID 19 pandemic.

Methods: Retrospective observational study. The virtual consultations made from PC (47 centres) to Rheumatology during 2020 were analysed. They were carried out through a computer programme, using the "Andalusian Health Service Virtual Consultation Platform" tool. A specific agenda was established for virtual consultations. The reason for the referral and the rheumatologist's diagnosis were collected. The response given to the PC was divided into four models: NON-TRIBUTARY (not related to the speciality), DISCHARGE (a diagnosis and therapeutic response is concluded), APPOINTMENT FOR CONSULTATION and FOLLOW-UP (new contact is requested, completing the information). The reasons for consultation, diagnoses, time and type of response were analysed. Results: 47 virtual consultations were carried out. 54.5\% (n 298) were closed as DISCHARGE. 27.4\% (n 150) were APPOINTMENT FOR CONSULTATION, and $17.7 \%$ (n 97) indicated FOLLOW-UP. Only $0.4 \%$ (n 2) were NOT TRIBUTARY

The average response time was 2 days 15 hours and $56 \mathrm{~min}$.

The most frequent reason for consultation was polyarthralgias (26.7\%, n 146) and after the rheumatologist's assessment a diagnosis was established in $89 \%$ of them. Inflammatory arthropathy accounted for $30.8 \%$ (n 45), osteoarthritis for $19.9 \%$ (n 29 ), fibromyalgia for $12.3 \%$ (n 18), polymyalgia rheumatica (PMR) for $6.9 \%$ (n 10), osteoporosis for $2.7 \%$ (n 4$)$ and connective tissue disease for $2.1 \%(n 3)$.

Another frequent reason for consultation was osteoporosis $(13.5 \% n 74)$, of which $85.1 \%$ ( $n$ 63) had a confirmed diagnosis and/or need for revision.

A diagnosis could be made via telematics in $89.6 \%$ of the consultations. $15.5 \%$ were osteoporosis (n 85), 14.9\% osteoarthritis ( $n$ 81), 10.5\% soft tissue injuries, $8.8 \%$ mechanical/ nonspecific pain (n 47), 7.1\% rheumatoid arthritis (n 39), 6.5\% fibromyalgia (n 34), 6.2\% connective tissue disease (n 34), 5.7\% PMR (n 31), 4.9\% suspected spondyloarthritis (n 26 ), $4.2 \%$ psoriatic arthritis ( $n 23$ ) and $4.2 \%$ microcrystalline arthritis ( $n 23$ ).

$27.4 \%$ ( $n$ 150) of the virtual consultations were required for assessment in a face-to-face appointment. We analysed the distribution over time (Figure 1). In the COVID 19 confinement phase (14 March - 21 June), the number of consultations increased, peaking in June, a behaviour that has persisted in the other mobility phases (October/November).

\section{Figure 1. ANNUAL PROGRESS OF VIRTUAL CONSULTATIONS TO RHEUMATOLOGY}
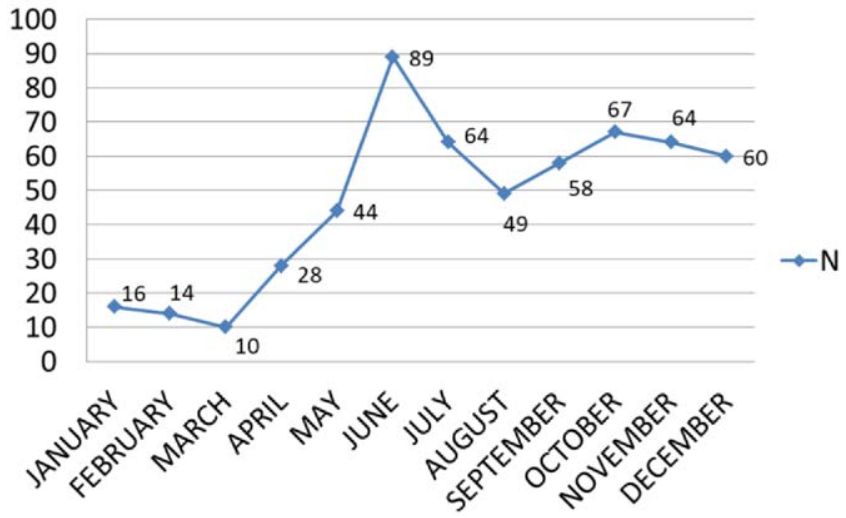

Conclusion: More than half of the virtual consultations carried out were resolved without face-to-face assessment, with a diagnosis being established in almost $90 \%$. It is an effective tool for rapid access to Rheumatology, detecting pathology requiring preferential attention, with a face-to-face appointment, as well as for the early diagnosis of inflammatory arthropathy, which was detected in a quarter of the consultations, as well as for the diagnosis and follow-up of osteoporosis. Virtual consultation facilitates a quick response, playing an even more relevant role in the current SARS CoV-2 pandemic situation. REFERENCES:

[1] B. Tejera, S. Bustabad. A new form of communication between rheumatology and primary care: The virtual consultation. Reum Clin., 12 (2016), pp 11-14 Disclosure of Interests: None declared

DOI: 10.1136/annrheumdis-2021-eular.1443

\section{POS1481-HPR SEXUAL EXPERIENCE IN MALE PATIENTS WITH ANKYLOSING SPONDYLITIS: RESULTS FROM A CROSS-SECTIONAL STUDY OF 113 PATIENTS}

L. $\mathrm{Hu}^{1}$, D. Gao ${ }^{1}, \mathrm{Y} . \mathrm{MA}^{1}$, Y. Wang ${ }^{1}, \mathrm{X} . \mathrm{Ji}^{1}$, F. Huang ${ }^{1} .{ }^{1}$ Chinese PLA General Hospital, Rheumatology, Beijing, China

Background: The expression and experience of sexuality is a key part of an individual self-identity ${ }^{1}$, so it is essential for both healthy individuals and patients.
Patients with ankylosing spondylitis (AS) may be susceptible to sexual issues due to disease activity, dysfunction and comorbid emotional problems. However, sexuality, especially sexual experience, are rarely paid attention in patients with

Objectives: Our study aims to assess sexual experience in male patients with AS, and analyze the factors affecting sexual experience.

Methods: This is a cross-sectional study. A total of 113 patients with AS and 46 healthy people were investigated, matched according to age and body mass index. The Sexual Experience Questionnaire is used to assess male sexua experience. Linear regression analysis is used to explore the contributions of clinical variables to worse sexual experience.

Results: There is a significant difference in the total sexual experience score between AS patients and healthy controls $(41.92 \pm 8.83$ vs $46.98 \pm 8.10, P=0.0013)$ Also, patients with AS have a worse score in all dimensions of sexual experience, including erectile function, individual satisfaction and couple satisfaction, comparing to healthy people. In the regression model after controlling for the effects of age, disease duration and body mass index, disease activity (BASDAI), function (BASFI), mobility (BASMI, chest expansion and finger-floor distance), health index (ASAS HI), sleep quality (PSQI) and psychological status (HADS HADS-A and HADS-D) are significant determinants of sexual experience, including erectile function (except for chest expansion), individual satisfaction (excep for BASMI) and couple satisfaction (except for BASMI). See Table 1 for details.

Table 1. Multivariable regression analysis of association between sexual experience and clinical outcomes

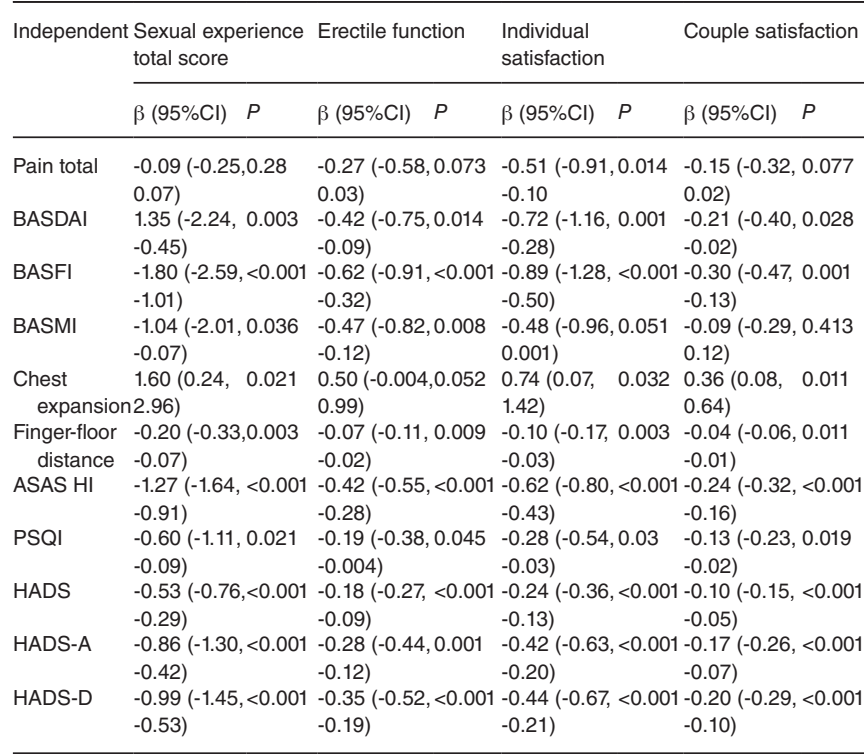

Conclusion: Worse sexual experience is associated with increased disease activity, decreased function, poor mobility, decreased health index, poor sleep quality and psychological status. Therefore, special attention to worse sexua experience in patients with AS is essential to assess disease-related suffering and develop new patient management strategies.

REFERENCES:

[1] Hill J, Bird H, Thorpe R. Effects of rheumatoid arthritis on sexual activity and relationships. Rheumatology (Oxford), 2003. 42(2):280-6.

Disclosure of Interests: None declared

DOI: 10.1136/annrheumdis-2021-eular.1470

\section{POS1482-HPR PAIN CATASTROPHIZING IS ASSOCIATED WITH RESIDUAL PAIN AFTER REACHING IMPROVED CONDITIONS OF SWOLLEN/TENDER JOINTS AND SERUM C-REACTIVE PROTEIN LEVEL}

T. Yoshida ${ }^{1,2}$, M. Hashimoto ${ }^{3}$, K. Murakami ${ }^{4}$, K. Murata $^{3,5}$, K. Nishitani $^{3,5}$ R. Watanabe ${ }^{3}$, T. Koyama ${ }^{1}$, R. Uehara ${ }^{1}$, M. Tanaka ${ }^{3}$, H. Ito ${ }^{3,5}$, S. Matsuda6. ${ }^{1}$ Kyoto Prefectural University of Medicine, Department of Epidemiology for Community Health and Medicine, Kyoto, Japan; ${ }^{2}$ Kyoto Prefectural University of Medicine, Department of Nursing, Kyoto, Japan; ${ }^{3}$ Graduate School of Medicine, Kyoto University, Department of Advanced Medicine for Rheumatic Diseases, Kyoto, Japan; ${ }^{4}$ Graduate School of Medicine, Kyoto University, Department of Rheumatology and Clinical Immunology, Kyoto, Japan; ${ }^{5}$ Graduate School of Medicine, Kyoto University, Department of Orthopaedic Surgery, Kyoto, Japan; ${ }^{5}$ Graduate School of Medicine, Kyoto University, Department of Orthopaedic Surgery, Kyoto, Japan 